\title{
1-LOOP EFFECTIVE ACTION ON THE 4-BALL
}

\author{
Giampiero Esposito ${ }^{1,2}$, Alexander Yu Kamenshchik ${ }^{3}$ and Giuseppe Pollifrone ${ }^{4}$ \\ ${ }^{1}$ Istituto Nazionale di Fisica Nucleare, Sezione di Napoli, \\ Mostra d'Oltremare Padiglione 20, 80125 Napoli, Italy \\ ${ }^{2}$ Dipartimento di Scienze Fisiche, \\ Mostra d'Oltremare Padiglione 19, 80125 Napoli, Italy \\ ${ }^{3}$ Nuclear Safety Institute, Russian Academy of Sciences, \\ Bolshaya Tulskaya 52, Moscow 113191, Russia \\ ${ }^{4}$ Dipartimento di Fisica, Università di Roma "La Sapienza", \\ and INFN, Sezione di Roma, Piazzale Aldo Moro 2, 00185 Roma, Italy
}

\begin{abstract}
This paper applies $\zeta$-function regularization to evaluate the 1-loop effective action for scalar field theories and Euclidean Maxwell theory in the presence of boundaries. After a comparison of two techniques developed in the recent literature, vacuum Maxwell theory is studied and the contribution of all perturbative modes to $\zeta^{\prime}(0)$ is derived: transverse, longitudinal and normal modes of the electromagnetic potential, jointly with ghost modes. The analysis is performed on imposing magnetic boundary conditions, when the Faddeev-Popov Euclidean action contains the particular gauge-averaging term which leads to a complete decoupling of all perturbative modes. It is shown that there is no cancellation of the contributions to $\zeta^{\prime}(0)$ resulting from longitudinal, normal and ghost modes.
\end{abstract}

PACS numbers: 0370, 0460 


\section{Introduction}

The approach to quantum field theory and quantum gravity in terms of the effective action has led to many deep insights into the structure of physical theories [1-8]. Over the last few years, a number of papers appeared in which the 1-loop effective action was calculated on non-trivial background geometries, including those with boundaries [7-12]. Here we carry out calculations of such a kind on the 4-ball for scalar fields and for Euclidean Maxwell theory, taking into account in the last case also the contributions of ghost and gauge modes.

Now we would like to recall the basic definitions concerning the effective action. For simplicity, we describe in this section the basic equations and ideas in the absence of gauge groups. The starting point is a functional-integral representation of the $<$ out $\mid$ in $>$ amplitude as [5]

$$
<\text { out } \mid \text { in }>=e^{i W[J]}=N \int e^{i\left(S[\varphi]+J_{l} \varphi^{l}\right)} \mu[\varphi] d \varphi
$$

where the integral is over all superclassical fields satisfying the given boundary conditions, $S[\varphi]$ is the classical action supplemented by boundary terms appropriate to the in and out eigenvectors, $J_{i}$ are external sources coupled to the field operators, and $\mu[\varphi]$ is a suitable measure for integration [5]. One may then define the effective action as the Legendre transform of $W[J]$ as

$$
\Gamma[\bar{\varphi}] \equiv W[J]-J_{l} \bar{\varphi}^{l}
$$

This action obeys the functional equation [5]

$$
\Gamma_{, i}[\bar{\varphi}]=-J_{i}
$$




\section{1-loop effective action on the 4-ball}

and an equivalent formulation of the theory may be based on the two equations [5]

$$
\begin{gathered}
e^{i \Gamma[\bar{\varphi}]}=N \int \exp i\left\{S[\varphi]+\Gamma_{, l}[\bar{\varphi}]\left(\bar{\varphi}^{l}-\varphi^{l}\right)\right\} \mu[\varphi] d \varphi \\
<A[\varphi]>=N e^{-i \Gamma[\bar{\varphi}]} \int A[\varphi] \exp i\left\{S[\varphi]+\Gamma_{, l}[\bar{\varphi}]\left(\bar{\varphi}^{l}-\varphi^{l}\right)\right\} \mu[\varphi] d \varphi
\end{gathered}
$$

where $<A[\varphi]>$ is the standard notation for chronological averages $[3,5]$. Everything in the theory can indeed be derived from (1.4) and (1.5), provided that ${ }_{i,} \Gamma_{, j}[\bar{\varphi}]$ is a non-singular integro-differential operator, which ensures that $\bar{\varphi}^{i}$ coincides with the chronological average of $\varphi^{i}$. Moreover, by paying attention to the measure, the chronological average of the operator field equations is found to be [5]

$$
<S_{, j}[\varphi]-i(\log \mu[\varphi])_{, j}>-\Gamma_{, j}[\bar{\varphi}]=0 .
$$

The extension to gauge fields and gravitation may be found, for example, in [3-6], and it leads to a powerful and elegant formulation of perturbative quantum field theory.

In recent years, as already mentioned above, motivated by the analysis of semi-classical effects in quantum field theory, impressive progress has been made in the calculation of the 1-loop effective action [7-12]. In the geometric approach [7], the 1-loop approximation is obtained after a careful application of the Schwinger-DeWitt method [1, 2, 13], and the information on the non-local part of the 1-loop effective action is encoded in the form factors (see $[7,8]$ and references therein). In the analytic approach, which has been successful in the more difficult case of manifolds with boundary, one applies $\zeta$-function regularization, the uniform asymptotic expansions of Bessel functions for the flat-space 


\section{1-loop effective action on the 4-ball}

case, or Legendre functions for the 4-sphere case, and suitable contour formulae to evaluate functional determinants for scalar, spinor and gauge fields [10-12].

For a given elliptic operator $\mathcal{A}$, the corresponding zeta-function $\zeta_{\mathcal{A}}$ is defined as the trace of its complex power $\mathcal{A}^{-s}$, and admits an analytic continuation to the whole complex$s$ plane as a meromorphic function [14]. It is then possible to make sense of $\zeta_{\mathcal{A}}(0)$ and $\zeta_{\mathcal{A}}^{\prime}(0)$, and the 1-loop effective action in four dimensions takes the form $[7,8]$

$$
\Gamma_{\mathcal{A}}^{(1)}=-\frac{1}{2} \zeta_{\mathcal{A}}^{\prime}(0)-\frac{1}{2} \zeta_{\mathcal{A}}(0) \log \left(\mu^{2}\right) .
$$

In physical applications, $\mathcal{A}$ is the Laplace operator, or the squared Dirac operator, or a matrix of elliptic operators in the case of gauge fields and gravitation, where gauge and ghost modes also contribute to the full 1-loop effective action.

In the analysis of manifolds with boundary, complete results for $\zeta^{\prime}(0)$ have been obtained in [9-12] for scalar and spin-1/2 fields, but for gauge fields and gravitation only the contributions of physical degrees of freedom (i.e. transverse or transverse-traceless modes) were obtained in $[11,12,15-17]$. The aim of our paper has been therefore to complete the recent investigations of the 1-loop effective action for gauge fields in the presence of boundaries. Since the calculations are, in general, extremely lengthy (at least if one wants to double-check them by hand, which remains a necessary step), we have focused on the simplest (but non-trivial) model, i.e. Euclidean Maxwell theory in the presence of boundaries [18-21]. Attention is focused on the particular gauge-averaging functional studied in [21], since this was found to lead to a trace anomaly which agrees with the one resulting from the transverse modes only [22], on imposing magnetic boundary conditions. It was 


\section{1-loop effective action on the 4-ball}

then necessary to understand whether the cancellation of the contributions of gauge and ghost modes in this gauge is also a property of the full 1-loop effective action.

For this purpose, section 2 describes the application of the algorithm by Bordag et al. [10] to four-dimensional Euclidean backgrounds with boundary. Section 3 studies instead the Barvinsky-Kamenshchik-Karmazin-Mishakov technique [15-17] in the case of Dirichlet boundary conditions. Section 4 obtains the 1-loop effective action with magnetic boundary conditions. As we said before, the gauge chosen is the one first derived in [21], where all perturbative modes for the electromagnetic potential and its ghost are decoupled. Concluding remarks and open problems are presented in section 5, and relevant details are given in the appendix.

\section{Bordag-Geyer-Kirsten-Elizalde algorithm}

A powerful analytic algorithm for the calculation of $\zeta^{\prime}(0)$ for manifolds with boundary is the one developed by Bordag et al. in [10]. We here describe its application to the analysis of the Laplace operator acting on massless scalar fields in flat four-dimensional Euclidean space bounded by a 3-sphere of radius $a$, in the case of Dirichlet boundary conditions. This is indeed the background considered in the original calculations of the following sections, and in $[18-21]$.

The work of [10] shows that the $\zeta$-function of such a four-dimensional boundary-value problem may be expressed as the sum of two terms as

$$
\zeta(s)=\sum_{l=0}^{\infty}(l+1)^{2} Z_{l+1}(s)+\sum_{i=-1}^{3} A_{i}(s)
$$




\section{1-loop effective action on the 4-ball}

where, with the notation in the appendix, one has

$$
\begin{gathered}
Z_{l+1}(s)=\frac{\sin (\pi s)}{\pi} \int_{0}^{\infty} d z\left(\frac{z(l+1)}{a}\right)^{-2 s} \frac{\partial}{\partial z}\left[\log I_{l+1}((l+1) z)-(l+1) \eta\right. \\
\left.+\log \left(\sqrt{2 \pi(l+1)}\left(1+z^{2}\right)^{\frac{1}{4}}\right)-\sum_{n=1}^{3} \frac{D_{n}(t)}{(l+1)^{n}}\right] \\
A_{-1}(s)=\frac{1}{4 \sqrt{\pi}} \frac{a^{2 s}}{\Gamma(s)} \frac{\Gamma\left(s-\frac{1}{2}\right)}{s} \zeta_{R}(2 s-3) \\
A_{1}(s)=-\frac{1}{2} \frac{a^{2 s}}{\Gamma(s)} \zeta_{R}(2 s-1) \sum_{j=0}^{1} x_{1, j}(1+2 j) \frac{\Gamma\left(s+j+\frac{1}{2}\right)}{\Gamma\left(j+\frac{3}{2}\right)} \\
A_{3}(s)=-\frac{1}{2} \frac{a^{2 s}}{\Gamma(s)} \zeta_{R}(2 s+1) \sum_{j=0}^{3} x_{3, j}(3+2 j) \frac{\Gamma\left(s+j+\frac{3}{2}\right)}{\Gamma\left(j+\frac{5}{2}\right)} \\
A_{2}(s)=-\frac{1}{2} \frac{a^{2 s}}{\Gamma(s)} \zeta_{R}(2 s) \sum_{j=0}^{2} x_{2, j}(2 s+2 j) \frac{\Gamma(s+j+1)}{\Gamma(j+2)}
\end{gathered}
$$

The $\zeta^{\prime}(0)$ value is then obtained by differentiation at $s=0$ of (2.1), making use of (2.2)(2.7), of the identity [10]

$$
\begin{aligned}
Z_{l+1}^{\prime}(0) & =\log (\Gamma(l+2))+(l+1)-(l+1) \log (l+1) \\
& -\frac{1}{2} \log (2 \pi(l+1))+\sum_{n=1}^{3} \frac{D_{n}(1)}{(l+1)^{n}} \\
& =\int_{0}^{\infty} d t\left(\frac{1}{2}-\frac{1}{t}+\frac{1}{\left(e^{t}-1\right)}\right) \frac{e^{-t(l+1)}}{t}+\sum_{n=1}^{3} \frac{D_{n}(1)}{(l+1)^{n}}
\end{aligned}
$$


and of the equations (A.4)-(A.6) of the appendix. The resulting $\zeta^{\prime}(0)$ value is found to be $[10]$

$$
\zeta^{\prime}(0)=\frac{173}{30240}+\frac{1}{90} \log (2 / a)+\frac{1}{3} \zeta_{R}^{\prime}(-3)-\frac{1}{2} \zeta_{R}^{\prime}(-2)+\frac{1}{6} \zeta_{R}^{\prime}(-1)
$$

\section{Barvinsky-Kamenshchik-Karmazin-Mishakov technique}

With the notation described in detail in [15-17], and applied also in [19], the $\zeta^{\prime}(0)$ value in field theory takes the form

$$
\zeta^{\prime}(0)=I_{\mathrm{reg}}^{R}(M=\infty)-I^{R}(M=0)-\int_{0}^{\infty} d M^{2} \log \left(M^{2}\right) \frac{d I_{\mathrm{pole}}\left(M^{2}\right)}{d M^{2}}
$$

In the case of a real, massless scalar field subject to homogeneous Dirichlet conditions on the 3-sphere, the BKKM function of [15-17] reads

$$
I\left(M^{2}, s\right) \equiv \sum_{n=1}^{\infty} n^{2-2 s} \log f_{n}(n M a)
$$

where $f_{n}(n M a)=(M a)^{-n} I_{n}(n M a)$. Thus, with the notation of equation (A.2) of the appendix, one finds

$$
\begin{aligned}
I_{\text {pole }} & =\frac{1}{6} u_{1}^{3}(t)+\frac{1}{2} u_{3}(t)-\frac{1}{2} u_{1}(t) u_{2}(t) \\
& =\frac{\left(5525 t^{9}-9945 t^{7}+4779 t^{5}-375 t^{3}\right)}{11520}
\end{aligned}
$$

where $t \equiv 1 / \sqrt{1+z^{2} a^{2}}$. It is now useful to set $x \equiv M^{2} a^{2}$, and then re-express the integral in $(3.1)$ as

$$
\mathcal{A}=\log \left(a^{2}\right)\left(I_{\text {pole }}(\infty)-I_{\text {pole }}(0)\right)-\int_{0}^{\infty} \frac{d I_{\text {pole }}}{d x} \log (x) d x
$$




\section{1-loop effective action on the 4-ball}

The integral in (3.4) can be evaluated with the help of equations (A.27)-(A.31) of the appendix. These lead to

$$
\mathcal{A}=\log \left(a^{2}\right)\left(I_{\text {pole }}(\infty)-I_{\text {pole }}(0)\right)+\frac{1}{360} \log (2)+\frac{47}{30240}
$$

Moreover, by virtue of the uniform asymptotic expansion (A.2), one finds

$$
I_{\text {reg }}^{R}(M=\infty)=I_{\log } \log \left(a^{2}\right)-\frac{1}{2} \log (2 \pi) \zeta_{R}(-2) .
$$

The calculation of $I^{R}(0)$ makes it necessary to use the representation of Bessel functions in terms of hypergeometric functions, which implies

$$
I^{R}(0)=-\sum_{n=1}^{\infty} n^{2}(n \log (2)+\log \Gamma(n+1))
$$

Thus, Stirling's formula for the logarithm of the $\Gamma$ function (cf (A.7)), jointly with the careful treatment of the limit as $s \rightarrow 0$ resulting from (3.2), lead to [23]

$$
\begin{aligned}
I_{\text {reg }}^{R}(M=\infty)-I^{R}(0) & =I_{\log } \log \left(a^{2}\right)+\frac{1}{120} \log (2)-\zeta_{R}^{\prime}(-3)-\frac{1}{2} \zeta_{R}^{\prime}(-2)-\frac{1}{120} \\
& +\frac{1}{2} \sum_{n=1}^{\infty} n^{2-2 s} \sum_{k=1}^{\infty} \frac{B_{2 k}}{(2 k-1) k n^{2 k-1}} .
\end{aligned}
$$

In the double sum in (3.8), only the terms with $k=1$ and 2 have divergent behaviour, and should be treated separately. They contribute $-\frac{1}{144}-\frac{1}{360} \frac{1}{s}+\frac{1}{180} \gamma$, where $-\frac{1}{360} \frac{1}{s}$ should be excluded because it belongs to $I_{\text {pole }}\left(M^{2}\right)$. Moreover, the double infinite sum from $k=3$ to $\infty$ in (3.8) contributes $\frac{4}{3} \zeta_{R}^{\prime}(-3)+\frac{1}{6} \zeta_{R}^{\prime}(-1)+\frac{7}{360}-\frac{1}{180} \gamma$. Hence one finds (cf [10])

$$
\begin{aligned}
I_{\mathrm{reg}}^{R}(M=\infty)-I^{R}(0) & =I_{\log } \log \left(a^{2}\right)+\frac{1}{120} \log (2) \\
& +\frac{1}{3} \zeta_{R}^{\prime}(-3)-\frac{1}{2} \zeta_{R}^{\prime}(-2)+\frac{1}{6} \zeta_{R}^{\prime}(-1)+\frac{1}{240}
\end{aligned}
$$


and the $\zeta^{\prime}(0)$ value is finally obtained from (3.5) and (3.9) as

$$
\zeta^{\prime}(0)=\frac{173}{30240}+\zeta(0) \log \left(a^{2} / 4\right)+\frac{1}{3} \zeta_{R}^{\prime}(-3)-\frac{1}{2} \zeta_{R}^{\prime}(-2)+\frac{1}{6} \zeta_{R}^{\prime}(-1)
$$

bearing in mind that $[15-17] \zeta(0)=I_{\log }+I_{\text {pole }}(\infty)-I_{\text {pole }}(0)$, which equals $-\frac{1}{180}$ in our case.

\section{Magnetic boundary conditions for Euclidean Maxwell theory}

In this section we evaluate the 1-loop effective action for Euclidean Maxwell theory on the flat four-dimensional background bounded by a 3-sphere, on using the particular gauge averaging functional described in [21], which enables one to decouple all the modes from the beginning.

For this purpose, one expands the normal and tangential components of the electromagnetic potential on a family of 3 -spheres as

$$
\begin{gathered}
A_{0}(x, \tau)=\sum_{n=1}^{\infty} R_{n}(\tau) Q^{(n)}(x) \\
A_{k}(x, \tau)=\sum_{n=2}^{\infty}\left[f_{n}(\tau) S_{k}^{(n)}(x)+g_{n}(\tau) P_{k}^{(n)}(x)\right] \quad \text { for all } k=1,2,3
\end{gathered}
$$

where $Q^{(n)}(x), S_{k}^{(n)}(x), P_{k}^{(n)}(x)$ are scalar, transverse and longitudinal vector harmonics on $S^{3}$, respectively [24]. Within the framework of Faddeev-Popov formalism, on choosing the gauge-averaging functional as [21]

$$
\Phi_{P}(A) \equiv{ }^{(4)} \nabla^{\mu} A_{\mu}-\frac{2}{3} A_{0} \operatorname{Tr} K
$$


and by setting to 1 the parameter $\alpha$ occurring in the total Euclidean action [18], one eventually gets the regular solutions for normal and longitudinal components of the electromagnetic potential and the basis functions for the ghost (denoted by $\epsilon_{n}(\tau)$ )

$$
\begin{gathered}
g_{n}(\tau)=A I_{\nu}(M \tau) \\
R_{n}(\tau)=B \frac{1}{\tau} I_{\nu}(M \tau) \\
\epsilon_{n}(M \tau)=C I_{\nu}(M \tau)
\end{gathered}
$$

where $\nu \equiv \sqrt{n^{2}-1}$ and $A, B, C$ are constants. Now we impose magnetic boundary conditions at the 3 -sphere boundary of radius $a$. These set to zero at the boundary the tangential components of the electromagnetic potential, the gauge-averaging functional and hence the Faddeev-Popov ghost field $[18,19]$. They lead, in the gauge (4.3), to Dirichlet boundary conditions for $f_{n}, g_{n}$ and ghost modes, and to Robin boundary conditions for $R_{n}$ modes.

On imposing the boundary conditions described above, one obtains [21]

$$
I_{\nu}(M a)=0
$$

for $g_{n}$ and $\epsilon_{n}$, and

$$
I_{\nu}^{\prime}(M a)=0
$$

for $R_{n}$. Note that, since $R_{1}$ is proportional to $I_{0}(M \tau) / \tau$ in our gauge, the decoupled mode for normal photons has to vanish, to ensure regularity at the origin (see [21]). 


\section{1-loop effective action on the 4-ball}

First, we evaluate the contribution of $\epsilon_{1}$ to the 1-loop effective action. Since $\epsilon_{1}$ is proportional to $I_{0}(M \tau)$, the analysis in [21], jointly with the asymptotic expansion of $I_{0}$ at large argument, implies (by virtue of the ghost degeneracy)

$$
\zeta_{\epsilon_{1}}^{\prime}(0)=\frac{1}{2} \log \left(a^{2}\right)+\log (2 \pi)
$$

We now study the contributions of all modes for $n \geq 2$ to the 1-loop effective action (with the exception of the transverse modes, whose effect was evaluated in [11, 12], as shown below). Since the degeneracy of $\epsilon_{n}$ is $-2 n^{2}$, whereas that for $g_{n}$ and $R_{n}$ is $n^{2}$, and bearing in mind that the $R_{n}$ obey Robin boundary conditions with $u=0$ (cf [10]), one can write $\zeta(s)$ as

$$
\lim _{m \rightarrow 0} \sum_{n=2}^{\infty} n^{2} \frac{\sin (\pi s)}{\pi} \int_{\frac{m a}{\nu}}^{\infty} d z\left[\left(\frac{z \nu}{a}\right)^{2}-m^{2}\right]^{-s} \frac{\partial}{\partial z}\left[\log \frac{z \nu}{a} I_{\nu}^{\prime}(z \nu)-\log I_{\nu}(z \nu)\right]
$$

Note that $m$ is a mass parameter which differs from $M$ used so far. Following [10], by subtracting and adding the leading terms of the uniform asymptotic expansions of Bessel functions and their first derivatives, one finds

$$
\zeta_{n \geq 2}(s)=\lim _{m \rightarrow 0}\left(\sum_{n=2}^{\infty} n^{2} Z_{\nu}(s)+\widetilde{A}_{0}(s)+\sum_{i=1}^{3} \widetilde{A}_{i}(s)\right)
$$

where (see the appendix)

$$
\begin{aligned}
Z_{\nu}(s) & =\frac{\sin (\pi s)}{\pi} \int_{\frac{m a}{\nu}}^{\infty} d z\left[\left(\frac{z \nu}{a}\right)^{2}-m^{2}\right]^{-s} \frac{\partial}{\partial z}\left\{\log \left[\frac{z}{\left(1+z^{2}\right)^{1 / 2}} \frac{I_{\nu}^{\prime}(z \nu)}{I_{\nu}(z \nu)}\right]\right. \\
& \left.+\sum_{i=1}^{3} \frac{\left(D_{i}(t)-M_{i}(t, 0)\right)}{\nu^{i}}\right\}
\end{aligned}
$$




$$
\begin{gathered}
\widetilde{A}_{0}(s)=\sum_{n=2}^{\infty} n^{2} \widetilde{A}_{0}^{\nu}(s)=\sum_{n=2}^{\infty} n^{2} \frac{\sin (\pi s)}{\pi} \int_{\frac{m a}{\nu}}^{\infty} d z\left[\left(\frac{z \nu}{a}\right)^{2}-m^{2}\right]^{-s} \frac{\partial}{\partial z} \log \left(1+z^{2}\right)^{1 / 2} \\
\widetilde{A}_{i}(s)=\sum_{n=2}^{\infty} n^{2} \frac{\sin (\pi s)}{\pi} \int_{\frac{m a}{\nu}}^{\infty} d z\left[\left(\frac{z \nu}{a}\right)^{2}-m^{2}\right]^{-s} \frac{\partial}{\partial z}\left(\frac{\left(M_{i}(t, 0)-D_{i}(t)\right)}{\nu^{i}}\right) .
\end{gathered}
$$

Remarkably, in this case the total $A_{-1}$ vanishes, since the terms resulting from $\exp (\nu \eta)$ cancel each other (see the appendix).

On using the analyticity of $Z_{\nu}(s)$ in the neighbourhood of $s=0$, one finds the derivative $Z_{\nu}^{\prime}(0)$ as $(\mathrm{cf}[10])$

$$
Z_{\nu}^{\prime}(0)=-\left[\log \frac{z}{\left(1+z^{2}\right)^{1 / 2}} \frac{I_{\nu}^{\prime}(z \nu)}{I_{\nu}(z \nu)}+\sum_{i=1}^{3} \frac{\left(D_{i}(t)-M_{i}(t, 0)\right)}{\nu^{i}}\right]_{z=\frac{m a}{\nu}}
$$

and as $m \rightarrow 0$, this becomes (since $M_{i}(1,0)=D_{i}(1)$ )

$$
Z_{\nu}^{\prime}(0)=0
$$

Thus, the contribution of the first infinite sum in (4.10) vanishes.

Moreover, since $u=0$ (as stated above), and bearing in mind the polynomials $M_{i}(t, 0)$ and $D_{i}(t)$, the sum of the effects of the $\widetilde{A}_{i}$, for $i=1,2,3$, yields in the massless limit (see the end of the appendix)

$$
\lim _{m \rightarrow 0}\left[\frac{d}{d s} \sum_{i=1}^{3} \widetilde{A}_{i}(s)\right]_{s=0}=-\frac{319}{1260} .
$$

Last, following [10], one immediately finds

$$
\widetilde{A}_{0}^{\nu}(s)=\frac{1}{2} m_{2}^{-2 s} F_{1}\left(1 ; s ; 1 ;-\left(\frac{\nu}{m a}\right)^{2}\right)=\frac{1}{2} m^{-2 s}\left[1+\frac{\left(n^{2}-1\right)}{(m a)^{2}}\right]^{-s} .
$$




\section{1-loop effective action on the 4-ball}

To evaluate the effect of $\widetilde{A}_{0}(s)$, it is sufficient to pick out the coefficient of $s$ in its expansion in powers of $s$. One then deals with the infinite sum

$$
\Sigma_{1} \equiv \frac{1}{2} \log \left(a^{2}\right) \sum_{n=2}^{\infty} n^{2}-\frac{1}{2} \lim _{y \rightarrow 0} \sum_{n=2}^{\infty} n^{2-2 y} \log \left(n^{2}-1\right) .
$$

Within the framework of $\zeta$-function regularization, the first sum in (4.18) yields $-\frac{1}{2} \log \left(a^{2}\right)$, whilst the finite part of the second sum can be obtained after expressing $\left(n^{2}-1\right)$ as $(n-1)(n+1)$, and then taking the limit

$$
-\frac{1}{2} \lim _{y \rightarrow 0}\left(\sum_{n=1}^{\infty}(n+1)^{2-2 y} \log (n)+\sum_{n=3}^{\infty}(n-1)^{2-2 y} \log (n)\right) .
$$

One thus finds

$$
\lim _{m \rightarrow 0}\left[\frac{d}{d s} \widetilde{A}_{0}(s)\right]_{s=0}=\zeta_{R}^{\prime}(-2)+\zeta_{R}^{\prime}(0)+\frac{1}{2} \log (2)-\frac{1}{2} \log \left(a^{2}\right)
$$

In the formalism of section 3 , the result (4.19) reflects the contributions of $I^{R}(0)$ and $I_{\text {reg }}^{R}(\infty)$ to $\zeta^{\prime}(0)[15-17]$

Interestingly, the full $\zeta^{\prime}(0)$ value differs from the contribution of transverse modes by the amount

$$
\zeta^{\prime}(0)-\zeta_{T}^{\prime}(0)=-\frac{319}{1260}+\log (2)+\frac{1}{2} \log (\pi)+\zeta_{R}^{\prime}(-2)
$$

since $\zeta_{R}^{\prime}(0)=-\frac{1}{2} \log (2 \pi)$. Last, bearing in mind the full $\zeta(0)$ value $-77 / 180[21]$ and the contribution of the transverse modes $f_{n}$ to $\zeta^{\prime}(0)[11,12]$

$$
\zeta_{\mathrm{T}}^{\prime}(0)=-\frac{6127}{15120}-\frac{29}{45} \log (2)-\frac{77}{90} \log (a)-\log (\pi)+\frac{2}{3} \zeta_{R}^{\prime}(-3)-\zeta_{R}^{\prime}(-2)-\frac{5}{3} \zeta_{R}^{\prime}(-1)
$$


and combining (4.20) and (4.21), one can write the full 1-loop effective action for Euclidean Maxwell theory, in the gauge (4.3), as (see 1.7)

$$
\Gamma^{(1)}=\frac{77}{360} \log \left(\mu^{2} a^{2}\right)+\frac{1991}{6048}+\frac{1}{4} \log (\pi)-\frac{8}{45} \log (2)-\frac{1}{3} \zeta_{R}^{\prime}(-3)+\frac{5}{6} \zeta_{R}^{\prime}(-1) .
$$

\section{Concluding remarks}

First, our paper has compared two analytic techniques $[10,15-17]$ for the evaluation of $\zeta^{\prime}(0)$ for manifolds with boundary, showing that they agree. In section 4 , these techniques have been applied to the calculation of the 1-loop effective action for Euclidean Maxwell theory subject to magnetic boundary conditions. On studying the particular gauge condition (4.3), which leads to a complete decoupling of longitudinal, normal and ghost modes from the beginning, such contributions have been found to yield a non-vanishing contribution to the full $\zeta^{\prime}(0)$ value within the framework of Faddeev-Popov formalism. This result appears interesting, since it was found in [21] that the gauge condition (4.3) leads to a full $\zeta(0)$ value which actually coincides with the contribution of the transverse modes $f_{n}$, i.e. $\zeta(0)=\zeta_{T}(0)=-\frac{77}{180}[22]$.

In other words, the detailed calculations of section 4 add evidence in favour of longitudinal, normal and ghost modes playing an essential role in obtaining the correct form of 1-loop calculations [19-21, 25]. However, the problem remains to identify unambiguously the unphysical modes of the quantum theory [26]. As far as we know, our paper has presented the first mode-by-mode calculation of the 1-loop effective action for gauge

fields in the presence of boundaries, when all perturbative modes are taken into account (the work in $[11,12]$ only considered the contribution of the so-called physical degrees of 
freedom, i.e. the transverse part of the electromagnetic potential, or transverse-traceless perturbations for linearized gravity). The extension to a broader class of relativistic gauges for Euclidean Maxwell theory [18, 19, 21], or to higher-spin fields, cannot be treated by hand, since it involves the uniform asymptotic expansions of determinants of $2 \times 2$ and $4 \times 4$ matrices. However, such a task is accessible to modern computer programmes, and we are confident that it can be accomplished in the near future. This is the next natural step, since in covariant gauges the differential operators are minimal, and one also achieves a well defined $3+1$ decomposition of the background 4-geometry [19-21].

The corresponding geometric form of $\zeta^{\prime}(0)$ for gauge fields and gravitation in the presence of boundaries is, however, much more difficult to obtain, and requires a greater effort. We thus hope that the research described in our paper will provide the first step towards the completion of DeWitt's effective action programme within the framework of 1-loop quantum cosmology [27] and quantum gravity [28, 29].

\section{Acknowledgments}

We are much indebted to Klaus Kirsten for correspondence and for enlightening conversations on his work on functional determinants. The work of A Kamenshchik was partially supported by the Russian Foundation for Fundamental Researches through grant No 9602-1000, and by the Russian Research Project "Cosmomicrophysics". 


\section{Appendix}

In section 2 , the equation obeyed by the eigenvalues by virtue of the boundary conditions is

$$
I_{l+1}(\sqrt{\lambda} a)=0 \quad \text { for all } l=0,1,2, \ldots
$$

where $I_{\rho}$ denotes, as usual, the regular Bessel function of order $\rho$. The uniform asymptotic expansion of $I_{\rho}(\rho z)$ as $\rho \rightarrow \infty$ is given by [30]

$$
I_{\rho}(\rho z) \sim \frac{1}{\sqrt{2 \pi \rho}} \frac{e^{\rho \eta}}{\left(1+z^{2}\right)^{\frac{1}{4}}}\left[1+\sum_{k=1}^{\infty} \frac{u_{k}(t)}{\rho^{k}}\right]
$$

where $t \equiv 1 / \sqrt{1+z^{2}}, \eta \equiv \sqrt{1+z^{2}}+\log \left[z /\left(1+\sqrt{1+z^{2}}\right)\right]$, and the $u_{k}$ polynomials are the Debye polynomials described in [30]. The 1-loop analysis makes it necessary to consider $\log \left(I_{\rho}(\rho z)\right)[10,15-17]$, and hence it is useful to derive the asymptotic expansion

$$
\log \left[1+\sum_{k=1}^{\infty} \frac{u_{k}(t)}{\rho^{k}}\right] \sim \sum_{n=1}^{\infty} \frac{D_{n}(t)}{\rho^{n}}
$$

where

$$
\begin{gathered}
D_{1}(t)=\sum_{j=0}^{1} x_{1, j} t^{1+2 j}=\frac{1}{8} t-\frac{5}{24} t^{3} \\
D_{2}(t)=\sum_{j=0}^{2} x_{2, j} t^{2+2 j}=\frac{1}{16} t^{2}-\frac{3}{8} t^{4}+\frac{5}{16} t^{6} \\
D_{3}(t)=\sum_{j=0}^{3} x_{3, j} t^{3+2 j}=\frac{25}{384} t^{3}-\frac{531}{640} t^{5}+\frac{221}{128} t^{7}-\frac{1105}{1152} t^{9} .
\end{gathered}
$$




\section{1-loop effective action on the 4-ball}

The identity (2.8) relies on the integral representation of $\log \Gamma(z)$, i.e. $[10,23]$

$$
\log \Gamma(z)=\left(z-\frac{1}{2}\right) \log (z)-z+\frac{1}{2} \log (2 \pi)+\int_{0}^{\infty}\left(\frac{1}{2}-\frac{1}{t}+\frac{1}{\left(e^{t}-1\right)}\right) \frac{e^{-t z}}{t} d t
$$

The contribution of $(2.8)$ to $\zeta^{\prime}(0)$ is obtained by taking the infinite sum $\sum_{l=0}^{\infty}(l+1)^{2} Z_{l+1}^{\prime}(0)$. A double integration by parts, and then the use of the identity

$$
\sum_{l=0}^{\infty} e^{-l t}=\frac{1}{\left(1-e^{-t}\right)}
$$

lead to a sum of divergent contributions which add up to zero, plus the following term:

$$
\begin{aligned}
Z^{\prime}(0, z) & =\frac{1}{360} \int_{0}^{\infty} \frac{t^{z} e^{-t}}{\left(1-e^{-t}\right)} d t+\int_{0}^{\infty} \frac{t^{z-3} e^{-t}}{\left(1-e^{-t}\right)} d t \\
& -6 \int_{0}^{\infty} \frac{t^{z-4} e^{-t}}{\left(1-e^{-t}\right)} d t+\int_{0}^{\infty} \frac{t^{z} e^{-t}}{\left(1-e^{-t}\right)} \frac{d^{2}}{d t^{2}}\left(\frac{1}{t} \frac{e^{-t}}{\left(1-e^{-t}\right)}\right) d t
\end{aligned}
$$

where the parameter $z$ has been introduced to regularize the divergences of the calculation [10]. Thus, on using the identities $\left(\zeta_{H}\right.$ being the Hurwitz $\zeta$-function)

$$
\begin{gathered}
\int_{0}^{\infty} \frac{x^{\sigma-1} e^{-\omega x}}{\left(1-e^{-x}\right)} d x=\Gamma(\sigma) \zeta_{H}(\sigma, \omega) \\
\int_{0}^{\infty} \frac{x^{\sigma} e^{-(\omega+1) x}}{\left(1-e^{-x}\right)^{2}} d x=\Gamma(\sigma+1)\left[\zeta_{H}(\sigma, \omega)-\omega \zeta_{H}(\sigma+1, \omega)\right] \\
\int_{0}^{\infty} \frac{x^{\sigma+1} e^{-(\omega+2) x}}{\left(1-e^{-x}\right)^{3}} d x=\frac{1}{2} \Gamma(\sigma+2)\left[\zeta_{H}(\sigma, \omega)-(1+2 \omega) \zeta_{H}(\sigma+1, \omega)\right. \\
\left.+\omega(1+\omega) \zeta_{H}(\sigma+2, \omega)\right]
\end{gathered}
$$




$$
\begin{aligned}
& \int_{0}^{\infty} \frac{x^{\sigma+2} e^{-(\omega+3) x}}{\left(1-e^{-x}\right)^{4}} d x=\frac{1}{6} \Gamma(\sigma+3)\left[\zeta_{H}(\sigma, \omega)-3(1+\omega) \zeta_{H}(\sigma+1, \omega)\right. \\
& \left.+\left(2+6 \omega+3 \omega^{2}\right) \zeta_{H}(\sigma+2, \omega)-\omega\left(2+3 \omega+\omega^{2}\right) \zeta_{H}(\sigma+3, \omega)\right]
\end{aligned}
$$

one finds

$$
\begin{aligned}
Z^{\prime}(0, z) & =\frac{1}{360} \Gamma(z+1) \zeta_{R}(z+1)+\left[\frac{1}{3} z^{3}-z^{2}+\frac{2}{3} z-8\right] \Gamma(z-3) \zeta_{R}(z-3) \\
+ & {\left[-\frac{1}{2} z^{2}+\frac{1}{2} z\right] \Gamma(z-2) \zeta_{R}(z-2)+\frac{1}{6}(z-1) \Gamma(z-1) \zeta_{R}(z-1) }
\end{aligned}
$$

At this stage, one can insert into (A.14) and (2.3)-(2.7) the expansions (as $\varepsilon \rightarrow 0$ )

$$
\begin{gathered}
\Gamma(\varepsilon-3)=-\frac{1}{6} \frac{1}{\varepsilon}+\frac{1}{6} \gamma-\frac{11}{36}+\mathrm{O}(\varepsilon) \\
\Gamma(\varepsilon-2)=\frac{1}{2} \frac{1}{\varepsilon}-\frac{1}{2} \gamma+\frac{3}{4}+\mathrm{O}(\varepsilon) \\
\Gamma(\varepsilon-1)=-\frac{1}{\varepsilon}+\gamma-1+\mathrm{O}(\varepsilon) \\
\Gamma(\varepsilon)=\frac{1}{\varepsilon}-\gamma+\mathrm{O}(\varepsilon) \\
\Gamma\left(\varepsilon-\frac{1}{2}\right)=-2 \sqrt{\pi}\left[1+\varepsilon(-\gamma-2 \log (2)+2)+\mathrm{O}\left(\varepsilon^{2}\right)\right] \\
\zeta_{R}(1+\varepsilon)=\frac{1}{\varepsilon}+\gamma+\mathrm{O}(\varepsilon) \\
\zeta_{R}(-n+\varepsilon)=\zeta_{R}(-n)+\varepsilon \zeta_{R}^{\prime}(-n)+\mathrm{O}\left(\varepsilon^{2}\right)
\end{gathered}
$$

and the identities [30]

$$
\zeta_{H}(s, 1)=\zeta_{R}(s)
$$




$$
\zeta_{H}\left(s, \frac{1}{2}\right)=\left(2^{s}-1\right) \zeta_{R}(s) .
$$

This leads to the result (2.9) for Dirichlet boundary conditions.

For Robin boundary conditions, we refer the reader to section 4 of [10], bearing in mind that the polynomials (A.4)-(A.6) are replaced by new polynomials $M_{n}(t, u)$ given by $[10,31]$

$$
\begin{gathered}
M_{1}(t, u)=\left(-\frac{3}{8}+u\right) t+\frac{7}{24} t^{3} \\
M_{2}(t, u)=\left(-\frac{3}{16}+\frac{1}{2} u-\frac{1}{2} u^{2}\right) t^{2}+\left(\frac{5}{8}-\frac{1}{2} u\right) t^{4}-\frac{7}{16} t^{6} \\
M_{3}(t, u)=\left(-\frac{21}{128}+\frac{3}{8} u-\frac{1}{2} u^{2}+\frac{1}{3} u^{3}\right) t^{3}+\left(\frac{869}{640}-\frac{5}{4} u+\frac{1}{2} u^{2}\right) t^{5} \\
+\left(-\frac{315}{128}+\frac{7}{8} u\right) t^{7}+\frac{1463}{1152} t^{9}
\end{gathered}
$$

where $u$ is the dimensionless parameter occurring in the formula for Robin boundary conditions [10]. In our section $4, u$ vanishes.

In section 3 , the integral in (3.4) is evaluated by means of the general formula

$$
\int_{0}^{\infty} \frac{\log (x)}{(x+1)^{m+1 / 2}} d x=\frac{2}{(2 m-1)}\left(2 \log (2)-\sum_{k=1}^{m-2} \frac{1}{k}-2 \sum_{k=m-1}^{2 m-3} \frac{1}{k}\right)
$$

which leads, in particular, to

$$
\begin{aligned}
& \int_{0}^{\infty} \frac{\log (x)}{(x+1)^{5 / 2}} d x=\frac{4}{3} \log (2)-\frac{4}{3} \\
& \int_{0}^{\infty} \frac{\log (x)}{(x+1)^{7 / 2}} d x=\frac{4}{5} \log (2)-\frac{16}{15}
\end{aligned}
$$


1-loop effective action on the 4-ball

$$
\begin{gathered}
\int_{0}^{\infty} \frac{\log (x)}{(x+1)^{9 / 2}} d x=\frac{4}{7} \log (2)-\frac{92}{105} \\
\int_{0}^{\infty} \frac{\log (x)}{(x+1)^{11 / 2}} d x=\frac{4}{9} \log (2)-\frac{704}{945}
\end{gathered}
$$

In section 4 , the $Z_{\nu}(s)$ and the $\widetilde{A}_{i}(s)$, for $i=-1, \ldots, 3$ are the sum of the contributions resulting from $g_{n}, R_{n}$ and $\epsilon_{n}$ with their own degeneracies. For the $g_{n}$ and $\epsilon_{n}$ modes (obeying Dirichlet boundary conditions) one has [10, 32]

$$
\begin{gathered}
Z_{\nu}^{D}(s)=\frac{\sin (\pi s)}{\pi} \int_{\frac{m a}{\nu}}^{\infty} d z\left[\left(\frac{z \nu}{a}\right)^{2}-m^{2}\right]^{-s} \frac{\partial}{\partial z}\left\{\log I_{\nu}(z \nu)\right. \\
\left.-\log \left[\frac{1}{\sqrt{2 \pi \nu}} \frac{e^{\nu \eta}}{\left(1+z^{2}\right)^{\frac{1}{4}}}\right]-\sum_{k=1}^{3} \frac{D_{k}(t)}{\nu^{k}}\right\} \\
A_{-1}^{\nu, D}=\frac{\sin (\pi s)}{\pi} \int_{\frac{m a}{\nu}}^{\infty} d z\left[\left(\frac{z \nu}{a}\right)^{2}-m^{2}\right]^{-s} \frac{\partial}{\partial z} \log \left(\frac{z^{-\nu}}{\sqrt{2 \pi \nu}} e^{\nu \eta}\right) \\
A_{0}^{\nu, D}=\frac{\sin (\pi s)}{\pi} \int_{\frac{m a}{\nu}}^{\infty} d z\left[\left(\frac{z \nu}{a}\right)^{2}-m^{2}\right]^{-s} \frac{\partial}{\partial z} \log \left(1+z^{2}\right)^{-\frac{1}{4}} \\
A_{i}^{\nu, D}=\frac{\sin (\pi s)}{\pi} \int_{\frac{m a}{\nu}}^{\infty} d z\left[\left(\frac{z \nu}{a}\right)^{2}-m^{2}\right]^{-s} \frac{\partial}{\partial z}\left(\frac{D_{i}(t)}{\nu^{i}}\right) .
\end{gathered}
$$

For the $R_{n}$ modes, which obey Robin boundary conditions with $u=0$, one has

$$
\begin{gathered}
Z_{\nu}^{R}(s)=\frac{\sin (\pi s)}{\pi} \int_{\frac{m a}{\nu}}^{\infty} d z\left[\left(\frac{z \nu}{a}\right)^{2}-m^{2}\right]^{-s} \frac{\partial}{\partial z}\left\{\log \left[\frac{z \nu}{a} I_{\nu}^{\prime}(z \nu)\right]\right. \\
\left.-\log \left[\frac{\nu}{a \sqrt{2 \pi \nu}} e^{\nu \eta}\left(1+z^{2}\right)^{\frac{1}{4}}\right]-\sum_{k=1}^{3} \frac{M_{k}(t, 0)}{\nu^{k}}\right\} \\
A_{-1}^{\nu, R}=A_{-1}^{\nu, D}
\end{gathered}
$$


1-loop effective action on the 4-ball

$$
A_{0}^{\nu, R}=-A_{0}^{\nu, D}
$$

and

$$
A_{i}^{\nu, R}=\frac{\sin (\pi s)}{\pi} \int_{\frac{m a}{\nu}}^{\infty} d z\left[\left(\frac{z \nu}{a}\right)^{2}-m^{2}\right]^{-s} \frac{\partial}{\partial z}\left(\frac{M_{i}(t, 0)}{\nu^{i}}\right) .
$$

The result (4.16) holds by virtue of the identities [32]

$$
\begin{gathered}
\int_{\frac{m a}{\nu}}^{\infty} d z\left[\left(\frac{z \nu}{a}\right)^{2}-m^{2}\right]^{-s} \frac{\partial}{\partial z} t^{l} \\
=-\frac{1}{2} m^{-2 s} \frac{l}{(m a)^{l}} \frac{\Gamma\left(s+\frac{1}{2} l\right) \Gamma(1-s)}{\Gamma\left(1+\frac{1}{2} l\right)} \nu^{l}\left[1+\left(\frac{\nu}{m a}\right)^{2}\right]^{-s-\frac{l}{2}} \\
\frac{\sin (\pi s)}{\pi} \Gamma(s) \Gamma(1-s)=1
\end{gathered}
$$

jointly with the formulae

$$
\begin{gathered}
M_{1}(t, 0)-D_{1}(t)=-\frac{1}{2} t+\frac{1}{2} t^{3} \\
M_{2}(t, 0)-D_{2}(t)=-\frac{1}{4} t^{2}+t^{4}-\frac{3}{4} t^{6} \\
M_{3}(t, 0)-D_{3}(t)=-\frac{11}{48} t^{3}+\frac{35}{16} t^{5}-\frac{67}{16} t^{7}+\frac{107}{48} t^{9}
\end{gathered}
$$

which result from (A.4)-(A.6) and (A.24)-(A.26). More precisely, denoting by $\tilde{x}_{l, j}$ the numerical coefficients occurring in (A.42)-(A.44), the insertion of (A.40) into (4.13) yields $(\mathrm{cf}(2.5)-(2.7))$

$$
\widetilde{A}_{1}(s)=-\frac{1}{2} \frac{a^{2 s}}{\Gamma(s)} \sum_{j=0}^{1} \tilde{x}_{1, j}(1+2 j) \frac{\Gamma\left(s+j+\frac{1}{2}\right)}{\Gamma\left(j+\frac{3}{2}\right)} \sum_{n=2}^{\infty} n^{2}\left(n^{2}-1\right)^{-s-\frac{1}{2}}
$$




$$
\begin{aligned}
& \widetilde{A}_{2}(s)=-\frac{1}{2} \frac{a^{2 s}}{\Gamma(s)} \sum_{j=0}^{2} \tilde{x}_{2, j}(2+2 j) \frac{\Gamma(s+j+1)}{\Gamma(j+2)} \sum_{n=2}^{\infty} n^{2}\left(n^{2}-1\right)^{-s-1} \\
& \widetilde{A}_{3}(s)=-\frac{1}{2} \frac{a^{2 s}}{\Gamma(s)} \sum_{j=0}^{3} \tilde{x}_{3, j}(3+2 j) \frac{\Gamma\left(s+j+\frac{3}{2}\right)}{\Gamma\left(j+\frac{5}{2}\right)} \sum_{n=2}^{\infty} n^{2}\left(n^{2}-1\right)^{-s-\frac{3}{2}} .
\end{aligned}
$$

These formulae make it necessary to use the expansion [17]

$$
\left(n^{2}-1\right)^{-s}=\sum_{r=0}^{\infty} \frac{\Gamma(r+s)}{\Gamma(s) \Gamma(r+1)} n^{-2 r-2 s}
$$

One can then insert (A.48) into (A.45)-(A.47), bearing in mind the expansions (A.18)(A.20) and the property $\sum_{j=0}^{l} \tilde{x}_{l, j}=0$, for all $l=1,2,3$, which holds by virtue of (A.42)(A.44). This implies that $\widetilde{A}_{1}(s)$ contributes $-\frac{1}{4}$ to $\zeta^{\prime}(0)$, which results from the expansion of $\zeta_{R}(2 s+1)$ in the identity

$$
\begin{aligned}
\sum_{n=2}^{\infty} n^{2}\left(n^{2}-1\right)^{-s-\frac{1}{2}} & =\zeta_{R}(2 s-1)-1+\left(s+\frac{1}{2}\right)\left(\zeta_{R}(2 s+1)-1\right) \\
& +\sum_{r=2}^{\infty} \frac{\Gamma\left(r+s+\frac{1}{2}\right)}{\Gamma\left(s+\frac{1}{2}\right) \Gamma(r+1)}\left[\zeta_{R}(2 r+2 s-1)-1\right]
\end{aligned}
$$

after insertion into (A.45). Moreover, $\widetilde{A}_{2}(s)$ yields a vanishing contribution, whilst $\widetilde{A}_{3}(s)$ contributes

$$
-\frac{1}{6}\left(2 \tilde{x}_{3,1}+\frac{16}{5} \tilde{x}_{3,2}+\frac{142}{35} \tilde{x}_{3,3}\right)=-\frac{1}{315}
$$

since $\sum_{n=2}^{\infty} n^{2}\left(n^{2}-1\right)^{-s-\frac{3}{2}}=\zeta_{R}(2 s+1)-1+\sum_{r=1}^{\infty} \frac{\Gamma\left(r+s+\frac{3}{2}\right)}{\Gamma\left(s+\frac{3}{2}\right) \Gamma(r+1)}\left[\zeta_{R}(2 r+2 s+1)-1\right]$, by virtue of (A.48). Hence one finds the value given in (4.16). 
In the formalism of section 3 , the same result is obtained by evaluating integrals of the kind

$$
J_{i} \equiv \int_{0}^{\infty} d z \log (z) \frac{\partial}{\partial z^{2}}\left(M_{i}(t, 0)-D_{i}(t)\right)
$$

and then using (A.27)-(A.31). For example, (A.27) and (A.28) imply that $J_{1}=1$. This contribution should be divided by 2 , bearing in mind that, as $n \rightarrow \infty, \frac{1}{\nu} \sim \frac{1}{n}\left(1+\frac{1}{2} \frac{1}{n^{2}}+\right.$ $\left.\mathrm{O}\left(n^{-4}\right)\right)$. Moreover, $J_{2}$ does not contribute to $\zeta^{\prime}(0)$, whilst $J_{3}=\frac{2}{315}$, and the result (4.16) is obtained, according to [15-17], as $-\frac{1}{2}\left(\frac{1}{2}+\frac{2}{315}\right)$.

\section{References}

[1] DeWitt B S 1965 Dynamical Theory of Groups and Fields (New York: Gordon and Breach)

[2] DeWitt B S 1975 Phys. Rep. 19295

[3] DeWitt B S 1984 in Relativity, Groups and Topology II eds B S DeWitt and R Stora (Amsterdam: North Holland)

[4] Vilkovisky G A 1984 Nucl. Phys. B 234125

[5] DeWitt B S 1987 in The Architecture of Fundamental Interactions at Short Distances eds P Ramond and R Stora (Amsterdam: North-Holland)

[6] Buchbinder I L, Odintsov S D and Shapiro I L 1992 Effective Action in Quantum Gravity (Bristol: IOP Publishing)

[7] Avramidi I G 1991 Nucl. Phys. B 355712 
1-loop effective action on the 4-ball

[8] Barvinsky A O, Gusev Yu V, Vilkovisky G A and Zhytnikov V V 1995 Nucl. Phys. B 439561

[9] Dowker J S and Apps J S 1995 Class. Quantum Grav. 121363

[10] Bordag M, Geyer B, Kirsten K and Elizalde E 1995 Zeta-function determinant of the Laplace operator on the D-dimensional ball HEP-TH 9505157 to appear in Comm. Math. Phys.

[11] Dowker J S 1995 Spin on the 4-ball HEP-TH 9508082

[12] Kirsten K and Cognola G 1995 Heat-kernel coefficients and functional determinants for higher-spin fields on the ball HEP-TH 9508088

[13] Schwinger J 1951 Phys. Rev. 82664

[14] Seeley R T 1967 Amer. Math. Soc. Proc. Symp. Pure Math. 10288

[15] Barvinsky A O, Kamenshchik A Yu, Karmazin I P and Mishakov I V 1992 Class. Quantum Grav. 9 L27

[16] Barvinsky A O, Kamenshchik A Yu and Karmazin I P 1992 Ann. Phys. (N.Y.) 219 201

[17] Kamenshchik A Yu and Mishakov I V 1992 Int. J. Mod. Phys. A 73713

[18] Esposito G 1994 Class. Quantum Grav. 11905

[19] Esposito G, Kamenshchik A Yu, Mishakov I V and Pollifrone G 1994 Class. Quantum Grav. 112939

[20] Esposito G and Kamenshchik A Yu 1994 Phys. Lett. 336B 324

[21] Esposito G, Kamenshchik A Yu, Mishakov I V and Pollifrone G 1995 Phys. Rev. D 522183 
1-loop effective action on the 4-ball

[22] Louko J 1988 Phys. Rev. D 38478

[23] Bateman H and Erdelyi A 1953 Higher Transcendental Functions Vol 1 (New York: McGraw-Hill)

[24] Lifshitz E M and Khalatnikov I M 1963 Adv. Phys. 12185

[25] Moss I G and Poletti S 1994 Phys. Lett. 333B 326

[26] Vassilevich D V 1995 Phys. Rev. D 52999

[27] Esposito G 1994 Quantum Gravity, Quantum Cosmology and Lorentzian Geometries (Lecture Notes in Physics m12) (Berlin: Springer)

[28] Barvinsky A O, Gusev Yu V, Zhytnikov V V and Vilkovisky G A 1995 Class. Quantum Grav. 122157

[29] Mirzabekian A G and Vilkovisky G A 1995 Class. Quantum Grav. 122173

[30] Abramowitz M and Stegun I A 1964 Handbook of Mathematical Functions with Formulas, Graphs and Mathematical Tables (New York: Dover)

[31] Moss I G 1989 Class. Quantum Grav. 6759

[32] Bordag M, Elizalde E and Kirsten K 1995 Heat-kernel coefficients of the Laplace operator on the D-dimensional ball HEP-TH 9503023 to appear in J. Math. Phys. 